\title{
Ideal class groups of cyclotomic number fields I
}

\author{
by
}

Franz LEMMERMEYER (Heidelberg)

1. Notation. Let $K \subset L$ be number fields; we will use the following notation:

- $\mathfrak{O}_{K}$ is the ring of integers of $K$;

- $E_{K}$ is its group of units;

- $W_{K}$ is the group of roots of unity contained in $K$;

- $w_{K}$ is the order of $W_{K}$;

- $\mathrm{Cl}(K)$ is the ideal class group of $K$;

- $[\mathfrak{a}]$ is the ideal class generated by the ideal $\mathfrak{a}$;

- $K^{1}$ denotes the Hilbert class field of $K$, that is the maximal abelian extension of $K$ which is unramified at all places;

- $j_{K \rightarrow L}$ denotes the transfer of ideal classes for number fields $K \subset L$, i.e. the homomorphism $\mathrm{Cl}(K) \rightarrow \mathrm{Cl}(L)$ induced by mapping an ideal $\mathfrak{a}$ to $\mathfrak{a} \mathfrak{O}_{L}$

- $\kappa_{L / K}$ denotes the capitulation kernel $\operatorname{ker} j_{K \rightarrow L}$;

Now let $K$ be a CM-field, i.e. a totally complex quadratic extension of a totally real number field; the following definitions are standard:

- $\sigma$ is complex conjugation;

- $K^{+}$denotes the maximal real subfield of $K$; this is the subfield fixed by $\sigma$;

- $\mathrm{Cl}^{-}(K)$ is the kernel of the map $N_{K / K^{+}}: \mathrm{Cl}(K) \rightarrow \mathrm{Cl}\left(K^{+}\right)$and is called the minus class group;

- $h^{-}(K)$ is the order of $\mathrm{Cl}^{-}(K)$, the minus class number;

- $Q(K)=\left(E_{K}: W_{K} E_{K^{+}}\right) \in\{1,2\}$ is Hasse's unit index.

We will need a well known result from class field theory. Assume that $K \subset L$ are CM-fields; then $\operatorname{ker}\left(N_{L / K}: \mathrm{Cl}(L) \rightarrow \mathrm{Cl}(K)\right)$ has order $\left(L \cap K^{1}: K\right)$. Since $K / K^{+}$is ramified at the infinite places, the norm $N_{K / K^{+}}: \mathrm{Cl}(K) \rightarrow \mathrm{Cl}\left(K^{+}\right)$is onto.

1991 Mathematics Subject Classification: Primary 11R18; Secondary 11R29. 
2. Hasse's unit index. Hasse's book $[\mathrm{H}]$ contains numerous theorems (Sätze 14-29) concerning the unit index $Q(L)=\left(E_{L}: W_{L} E_{K}\right)$, where $K=L^{+}$is the maximal real subfield of a cyclotomic number field $L$. Hasse considered only abelian number fields $L / \mathbb{Q}$, hence he was able to describe these fields in terms of their character groups $X(L)$; as we are interested in results on general CM-fields, we have to proceed in a different manner. But first we will collect some of the most elementary properties of $Q(L)$ (see also $[\mathrm{H}]$ and $[\mathrm{W}]$; a reference "Satz *" always refers to Hasse's book $[\mathrm{H}])$ in

Proposition 1. Let $K \subset L$ be $C M$-fields; then

(a) $(\operatorname{Satz} 14) Q(L)=\left(E_{L}: W_{L} E_{L^{+}}\right)=\left(E_{L}^{\sigma-1}: W_{L}^{2}\right)=\left(E_{L}^{\sigma+1}: E_{L^{+}}^{2}\right)$; in particular, $Q(L) \in\{1,2\}$;

(b) $($ Satz 16,17$)$ If $Q(L)=2$ then $\kappa_{L / L^{+}}=1$;

(c) (Satz 25) If $L^{+}$contains units with any given signature, then $Q(L)=1$

(d) (Satz 29) $Q(K) \mid Q(L) \cdot\left(W_{L}: W_{K}\right)$;

(e) (compare Satz 26) Suppose that $N_{L / K}: W_{L} / W_{L}^{2} \rightarrow W_{K} / W_{K}^{2}$ is onto. Then $Q(L) \mid Q(K)$;

(f) $([\mathrm{HY}$, Lemma 2]) If $(L: K)$ is odd, then $Q(L)=Q(K)$;

(g) (Satz 27) If $L=\mathbb{Q}\left(\zeta_{m}\right)$, where $m \not \equiv 2 \bmod 4$ is composite, then $Q(L)=2$;

(h) (see Example 4 below) Let $K_{1} \subseteq \mathbb{Q}\left(\zeta_{m}\right)$ and $K_{2} \subseteq \mathbb{Q}\left(\zeta_{n}\right)$ be abelian $C M$-fields, where $m=p^{\mu}$ and $n=q^{\nu}$ are prime powers such that $p \neq q$, and let $K=K_{1} K_{2}$; then $Q(K)=2$.

The proofs are straightforward:

(a) The map $\varepsilon \rightarrow \varepsilon^{\sigma-1}$ induces an epimorphism $E_{L} \rightarrow E_{L}^{\sigma-1} / W_{L}^{2}$. If $\varepsilon^{\sigma-1}=\zeta^{2}$ for some $\zeta \in W_{L}$, then $(\zeta \varepsilon)^{\sigma-1}=1$, and $\zeta \varepsilon \in E_{L^{+}}$. This shows that $\sigma-1$ gives rise to an isomorphism $E_{L} / W_{L} E_{L^{+}} \rightarrow E_{L}^{\sigma-1} / W_{L}^{2}$, hence we have $\left(E_{L}: W_{L} E_{L^{+}}\right)=\left(E_{L}^{\sigma-1}: W_{L}^{2}\right)$. The other claim is proved similarly.

(b) Since $W_{L} / W_{L}^{2}$ is cyclic of order 2, the first claim follows immediately from (a). Now let $\mathfrak{a}$ be an ideal in $\mathfrak{O}_{K}$ such that $\mathfrak{a} \mathfrak{O}_{L}=\alpha \mathfrak{O}_{L}$. Then $\alpha^{\sigma-1}=\zeta$ for some root of unity $\zeta \in L$, and $Q(L)=2$ shows that $\zeta=\varepsilon^{\sigma-1}$ for some $\varepsilon \in E_{L}$. Now $\alpha \varepsilon^{-1}$ generates $\mathfrak{a}$ and is fixed by $\sigma$, hence lies in $K$. This shows that $\mathfrak{a}$ is principal in $K$, i.e. that $\kappa_{L / L^{+}}=1$.

(c) Units in $L^{+}$which are norms from $L$ are totally positive; our assumption implies that totally positive units are squares, hence we get $E_{L}^{\sigma+1}=$ $E_{L^{+}}^{2}$, and our claim follows from (a).

(d) First note that $\left(W_{L}: W_{K}\right)=\left(W_{L}^{2}: W_{K}^{2}\right)$; then 


$$
\begin{aligned}
Q(L) \cdot\left(W_{L}: W_{K}\right) & =\left(E_{L}^{\sigma-1}: W_{L}^{2}\right)\left(W_{L}^{2}: W_{K}^{2}\right)=\left(E_{L}^{\sigma-1}: E_{K}^{\sigma-1}\right)\left(E_{K}^{\sigma-1}: W_{K}^{2}\right) \\
& =\left(E_{L}^{\sigma-1}: E_{K}^{\sigma-1}\right) \cdot Q(K)
\end{aligned}
$$

proves the claim.

(e) Since $Q(L)=2$, there is a unit $\varepsilon \in E_{L}$ such that $\varepsilon^{\sigma-1}=\zeta$ generates $W_{L} / W_{L}^{2}$. Taking the norm to $K$ shows that $\left(N_{L / K} \varepsilon\right)^{\sigma-1}=N_{L / K}(\zeta)$ generates $W_{K} / W_{K}^{2}$, i.e. we have $Q(K)=2$.

(f) If $(L: K)$ is odd, then $\left(W_{L}: W_{K}\right)$ is odd, too, and we get $Q(K) \mid Q(L)$ from (d) and $Q(L) \mid Q(K)$ from (e).

(g) In this case, $1-\zeta_{m}$ is a unit, and we find $\left(1-\zeta_{m}\right)^{1-\sigma}=-\zeta_{m}$. Since $-\zeta_{m} \in W_{L} \backslash W_{L}^{2}$, we must have $Q(L)=2$.

(h) First assume that $m$ and $n$ are odd. A subfield $F \subseteq L=\mathbb{Q}\left(\zeta_{m}\right)$, where $m=p^{\mu}$ is an odd prime power, is a CM-field if and only if it contains the maximal 2-extension contained in $L$, i.e. if and only if $(L: F)$ is odd. Since $\left(\mathbb{Q}\left(\zeta_{m}\right): K_{1}\right)$ and $\left(\mathbb{Q}\left(\zeta_{n}\right): K_{2}\right)$ are both odd, so is $\left(\mathbb{Q}\left(\zeta_{m n}\right): K_{1} K_{2}\right)$; moreover, $\mathbb{Q}\left(\zeta_{m n}\right)$ has unit index $Q=2$, hence the assertion follows from (f) and (g).

Now assume that $p=2$. If $\sqrt{-1} \in K_{1}$, then we must have $K_{1}=\mathbb{Q}\left(\zeta_{m}\right)$ for $m=2^{\alpha}$ and some $\alpha \geq 2$ (complex subfields of the field of $2^{\mu}$ th roots of unity containing $\sqrt{-1}$ necessarily have this form). Now $n$ is odd and $K_{2} \subseteq \mathbb{Q}\left(\zeta_{n}\right)$ is complex, hence $\left(\mathbb{Q}\left(\zeta_{n}\right): K_{2}\right)$ is odd. By (f) it suffices to show that $K_{1}\left(\zeta_{n}\right)=\mathbb{Q}\left(\zeta_{m n}\right)$ has unit index 2 , and this follows from (g).

If $\sqrt{-1} \notin K_{1}$, let $\widetilde{K}_{1}=K_{1}(i)$; then $\widetilde{K}_{1}=\mathbb{Q}\left(\zeta_{m}\right)$ for $m=2^{\alpha}$ and some $\alpha \geq 2$, and in the last paragraph we have seen that $Q\left(\widetilde{K}_{1} K_{2}\right)=2$. Hence we only need to show that the norm map

$$
N: W_{\widetilde{K}_{1}} / W_{\widetilde{K}_{1}}^{2} \rightarrow W_{K_{1}} / W_{K_{1}}^{2}
$$

is onto: since $\left(W_{\widetilde{K}_{1} K_{2}}: W_{\widetilde{K}_{1}}\right)$ is odd, this implies $2=Q\left(\widetilde{K}_{1} K_{2}\right) \mid Q\left(K_{1} K_{2}\right)$ by (e). But the observation that the non-trivial automorphism of $\mathbb{Q}\left(\zeta_{m}\right) / K_{1}$ maps $\zeta_{m}$ to $-\zeta_{m}^{-1}$ implies at once that $N\left(\zeta_{m}\right)=-1$, and -1 generates $W_{K_{1}} / W_{K_{1}}^{2}$.

Now let $L$ be a CM-field with maximal real subfield $K$; we will call $L / K$ essentially ramified if $L=K(\sqrt{\alpha})$ and there is a prime ideal $\mathfrak{p}$ in $\mathfrak{O}_{K}$ such that the exact power of $\mathfrak{p}$ dividing $\alpha \mathfrak{O}_{K}$ is odd; it is easily seen that this does not depend on which $\alpha$ we choose. Moreover, every ramified prime ideal $\mathfrak{p}$ above an odd prime $p$ is necessarily essentially ramified. We leave it as an exercise to the reader to verify that our definition of essential ramification coincides with Hasse's [H, Sect. 22]; the key observation is the ideal equation $(4 \alpha)=\mathfrak{a}^{2} \mathfrak{d}$, where $\mathfrak{d}=\operatorname{disc}(K(\sqrt{\alpha}) / K)$ and $\mathfrak{a}$ is an integral ideal in $\mathfrak{O}_{K}$.

We will also need certain totally real elements of norm 2 in the field of 
$2^{m}$ th roots of unity: to this end we define

$$
\begin{aligned}
\pi_{2} & =2=2+\zeta_{4}+\zeta_{4}^{-1}, \\
\pi_{3} & =2+\sqrt{2}=2+\zeta_{8}+\zeta_{8}^{-1}, \\
& \vdots \\
\pi_{n} & =2+\sqrt{\pi_{n-1}}=2+\zeta_{2^{n}}+\zeta_{2^{n}}^{-1} .
\end{aligned}
$$

Let $m \geq 2, L=\mathbb{Q}\left(\zeta_{2^{m+1}}\right)$ and $K=\mathbb{Q}\left(\pi_{m}\right)$; then $L / K$ is an extension of type $(2,2)$ with subfields $K_{1}=\mathbb{Q}\left(\zeta_{2^{m}}\right), K_{2}=\mathbb{Q}\left(\sqrt{\pi_{m}}\right)$ and $K_{3}=\mathbb{Q}\left(\sqrt{-\pi_{m}}\right)$. Moreover, $K_{2} / K$ and $K_{3} / K$ are essentially ramified, whereas $K_{1} / K$ is not.

Theorem 1. Let L be a CM-field with maximal real subfield $K$.

(i) If $w_{L} \equiv 2 \bmod 4$, then:

1. If $L / K$ is essentially ramified, then $Q(L)=1$, and $\kappa_{L / K}=1$.

2. If $L / K$ is not essentially ramified, then $L=K(\sqrt{\alpha})$ for some $\alpha \in \mathfrak{O}_{K}$ such that $\alpha \mathfrak{O}_{K}=\mathfrak{a}^{2}$, where $\mathfrak{a}$ is an integral ideal in $\mathfrak{O}_{K}$, and

(a) $Q(L)=2$ if $\mathfrak{a}$ is principal, and

(b) $Q(L)=1$ and $\kappa_{L / K}=\langle[\mathfrak{a}]\rangle$ if $\mathfrak{a}$ is not principal.

(ii) If $w_{L} \equiv 2^{m} \bmod 2^{m+1}$, where $m \geq 2$, then $L / K$ is not essentially ramified, and:

1. If $\pi_{m} \mathfrak{O}_{K}$ is not an ideal square, then $Q(L)=1$ and $\kappa_{L / K}=1$.

2. If $\pi_{m} \mathfrak{O}_{K}=\mathfrak{b}^{2}$ for some integral ideal $\mathfrak{b}$, then
(a) $Q(L)=2$ if $\mathfrak{b}$ is principal, and
(b) $Q(L)=1$ and $\kappa_{L / K}=\langle[\mathfrak{b}]\rangle$ if $\mathfrak{b}$ is not principal.

For the proof of Theorem 1 we will need the following

Lemma 1. Let $L=K(\sqrt{\pi})$, and let $\sigma$ denote the non-trivial automorphism of $L / K$. Moreover, let $\mathfrak{b}$ be an ideal in $\mathfrak{O}_{K}$ such that $\mathfrak{b} \mathfrak{O}_{L}=(\beta)$ and $\beta^{\sigma-1}=-1$ for some $\beta \in L$. Then $\pi \mathfrak{O}_{K}$ is an ideal square in $\mathfrak{O}_{K}$. If , on the other hand, $\beta^{\sigma-1}=\zeta$, where $\zeta$ is a primitive $2^{m}$ th root of unity, then $\pi_{m} \mathfrak{O}_{K}$ is an ideal square in $\mathfrak{O}_{K}$.

Proof. We have $(\beta \sqrt{\pi})^{\sigma-1}=1$, hence $\beta \sqrt{\pi} \in K$. Therefore $\mathfrak{b}$ and $\mathfrak{c}=(\beta \sqrt{\pi})$ are ideals in $\mathfrak{O}_{K}$, and $\left(\mathfrak{c} \mathfrak{b}^{-1}\right)^{2}=\pi \mathfrak{O}_{K}$ proves our claim.

Now assume that $\beta^{\sigma-1}=\zeta$; then $\sigma$ fixes $(1-\zeta) \beta^{-1}$, hence $((1-\zeta) \beta)$ and $\mathfrak{c}=(1-\zeta)=\mathfrak{c}^{\sigma}$ are ideals in $\mathfrak{O}_{K}$, and $\mathfrak{c}^{2}=N_{L / K}(1-\zeta)=\left(2+\zeta+\zeta^{-1}\right) \mathfrak{O}_{K}$ is indeed an ideal square in $\mathfrak{O}_{K}$ as claimed.

Proof of Theorem 1. (i) Assume that $w_{L} \equiv 2 \bmod 4$. 
Case 1: $L / K$ is essentially ramified. Assume we had $Q(L)=2$; then $E_{L}^{\sigma-1}=W_{L}$, hence there is a unit $\varepsilon \in E_{L}$ such that $\varepsilon^{\sigma-1}=-1$. Write $L=K(\sqrt{\pi})$, and apply Lemma 1 to $\mathfrak{b}=(1), \beta=\varepsilon$ : this will yield the contradiction that $L / K$ is not essentially ramified.

Case 2: $L / K$ is not essentially ramified. Then $L=K(\sqrt{\alpha})$ for some $\alpha \in \mathfrak{O}_{K}$ such that $\alpha \mathfrak{O}_{K}=\mathfrak{a}^{2}$, where $\mathfrak{a}$ is an integral ideal in $\mathfrak{O}_{K}$.

(a) If $\mathfrak{a}$ is principal, say $\mathfrak{a}=\beta \mathfrak{D}_{K}$, then there is a unit $\varepsilon \in E_{K}$ such that $\alpha=\beta^{2} \varepsilon$, and we see that $L=K(\sqrt{\varepsilon})$. Now $\sqrt{\varepsilon}^{\sigma-1}=-1$ is no square since $w_{L} \equiv 2 \bmod 4$, and Proposition 1 (a) gives $Q(L)=2$.

(b) If $\mathfrak{a}$ is not principal, then the ideal class [a] capitulates in $L / K$ because $\mathfrak{a} \mathfrak{O}_{L}=\sqrt{\alpha} \mathfrak{O}_{L}$. Proposition $1(\mathrm{~b})$ shows that $Q(L)=1$.

(ii) Assume that $w_{L} \equiv 2^{m} \bmod 2^{m+1}$ for some $m \geq 2$.

Case 1: Assume that $Q(L)=2$ or $\kappa_{L / K} \neq 1$. Then Lemma 1 says that $\pi_{m} \mathfrak{O}_{K}=\mathfrak{b}^{2}$ is an ideal square in $\mathfrak{O}_{K}$ contrary to our assumption.

Case 2: $\pi_{m}=\mathfrak{b}^{2}$ is an ideal square in $\mathfrak{O}_{K}$. If $\mathfrak{b}$ is not principal, then $\mathfrak{b} \mathfrak{O}_{L}=(1-\zeta)$ shows that $\kappa_{L / K}=\langle[\mathfrak{b}]\rangle$, and Proposition $1(\mathrm{~b})$ gives $Q(L)=1$. If, on the other hand, $\mathfrak{b}=\beta \mathfrak{O}_{K}$, then $\eta \beta^{2}=\pi_{m}$ for some unit $\eta \in E_{K}$. If $\eta$ were a square in $\mathfrak{O}_{K}$, then $\pi_{m}$ would also be a square, and $L=K(\sqrt{-1})$ would contain the $2^{m+1}$ th roots of unity. Now $\eta \beta^{2}=\pi_{m}=\zeta^{-1}(1+\zeta)^{2}$, hence $\eta \zeta$ is a square in $L$, and we have $Q(L)=2$ as claimed.

Rem ark. For $L / \mathbb{Q}$ abelian, Theorem 1 is equivalent to Hasse's Satz 22; we will again only sketch the proof: suppose that $w_{L} \equiv 2^{m} \bmod 2^{m+1}$ for some $m \geq 2$, and define $L^{\prime}=L\left(\zeta_{2^{m+1}}\right), K^{\prime}=L^{\prime} \cap \mathbb{R}$. Then $K^{\prime} / K$ is essentially ramified if and only if $\pi_{m}$ is not an ideal square in $\mathfrak{O}_{K}$ (because $K^{\prime}=K\left(\pi_{m+1}\right)=K\left(\sqrt{\pi_{m}}\right)$ ). The asserted equivalence should now be clear. Except for the results on capitulation, Theorem 1 is also contained in [O] (for general CM-fields).

EXAmples. 1. Complex subfields $L$ of $\mathbb{Q}\left(\zeta_{p^{m}}\right)$, where $p$ is prime, have unit index $Q(L)=1$ (Hasse's Satz 23) and $\kappa_{L / L^{+}}=1$ : since $p$ ramifies completely in $\mathbb{Q}\left(\zeta_{p^{m}}\right) / \mathbb{Q}, L / L^{+}$is essentially ramified if $p \neq 2$, and the claim follows from Theorem 1 . If $p=2$ and $L / L^{+}$is not essentially ramified, then we must have $L=\mathbb{Q}\left(\zeta_{2^{\mu}}\right)$ for some $\mu \in \mathbb{N}$, and we find $Q(L)=1$ by Theorem 1(ii.1).

2. $L=\mathbb{Q}\left(\zeta_{m}\right)$ has unit index $Q(L)=1$ if and only if $m \not \equiv 2 \bmod 4$ is a prime power (Satz 27). This follows from Example 1 and Proposition 1(e).

3. If $K$ is a CM-field, which is essentially ramified at a prime ideal $\mathfrak{p}$ above $p \in \mathbb{N}$, and if $F$ is a totally real field such that $p \nmid \operatorname{disc} F$, then $Q(L)=1$ and $\kappa_{L / L^{+}}=1$ for $L=K F$ : this is again due to the fact that either $L / L^{+}$is essentially ramified at the prime ideals above $\mathfrak{p}$, or $p=2$ and 
$K=K^{+}(\sqrt{-1})$. In the first case, we have $Q(L)=1$ by Theorem 1 (i.1), and in the second case by Theorem 1(ii.1).

4. Suppose that the abelian CM-field $K$ is the compositum $K=K_{1} \ldots K_{t}$ of fields with pairwise different prime power conductors; then $Q(K)=1$ if and only if exactly one of the $K_{i}$ is imaginary (Uchida [U, Prop. 3]). The proof is easy: if there is exactly one complex field among the $K_{j}$, then $Q(K)=1$ by Example 3 . Now suppose that $K_{1}$ and $K_{2}$ are imaginary; we know $Q\left(K_{1} K_{2}\right)=2$ (Proposition $1(\mathrm{~h})$ ), and from the fact that the $K_{j}$ have pairwise different conductors we deduce that $\left(W_{K}: W_{K_{1} K_{2}}\right) \equiv 1 \bmod 2$. Now the claim follows from Hasse's Satz 29 (Proposition 1(c)). Observe that $\kappa_{K / K^{+}}=1$ in all cases.

5. Cyclic extensions $L / \mathbb{Q}$ have unit index $Q(L)=1$ (Hasse's Satz 24): Let $F$ be the maximal subfield of $L$ such that $(F: \mathbb{Q})$ is odd. Then $F$ is totally real, and $2 \nmid \operatorname{disc} F$ (this follows from the theorem of Kronecker and Weber). Similarly, let $K$ be the maximal subfield of $L$ such that $(K: \mathbb{Q})$ is a 2-power: then $K$ is a CM-field, and $L=F K$. If $K / K^{+}$is essentially ramified at a prime ideal $\mathfrak{p}$ above an odd prime $p$, then so is $L / L^{+}$, because $L / \mathbb{Q}$ is abelian, and all prime ideals in $F$ have odd ramification index. Hence the claim in this case follows by Example 3 above.

If, however, $K / K^{+}$is not essentially ramified at a prime ideal $\mathfrak{p}$ above an odd prime $p$, then $\operatorname{disc} K$ is a 2-power (recall that $K / \mathbb{Q}$ is cyclic of 2-power degree). Applying the theorem of Kronecker and Weber, we find that $K \subseteq \mathbb{Q}(\zeta)$, where $\zeta$ is some primitive $2^{m}$ th root of unity. If $K / K^{+}$is essentially ramified at a prime ideal above 2 , then so is $L / L^{+}$, and Theorem 1 gives us $Q(L)=1$. If $K / K^{+}$is not essentially ramified at a prime ideal above 2 , then we must have $K=\mathbb{Q}(\zeta)$, where $\zeta$ is a primitive $2^{m}$ th root of unity; but now $\pi_{m} \mathfrak{O}_{L^{+}}$is not the square of an integral ideal, and we have $Q(L)=1$ by Theorem 1 . Alternatively, we may apply Proposition 1(e) and observe that $Q(K)=1$ by Example 1 .

6. Let $p \equiv 1 \bmod 8$ be a prime such that the fundamental unit $\varepsilon_{2 p}$ of $\mathbb{Q}(\sqrt{2 p})$ has norm +1 (by $[\mathrm{S}]$, there are infinitely many such primes; note also that $N \varepsilon_{2 p}=+1 \Leftrightarrow(2, \sqrt{2 p})$ is principal). Put $K=\mathbb{Q}(i, \sqrt{2 p})$ and $L=\mathbb{Q}(i, \sqrt{2}, \sqrt{p})$. Then $Q(K)=2$ by Theorem $1($ ii.2)(a), whereas the fact that $L$ is the compositum of $\mathbb{Q}\left(\zeta_{8}\right)$ and $\mathbb{Q}(\sqrt{p})$ shows that $Q(L)=1$ (Example 4). This generalization of Lenstra's example given by Martinet in $[\mathrm{H}]$ is contained in Theorem 4 of $[\mathrm{HY}]$, where several other results of this kind can be found.

3. Masley's theorem $h_{m}^{-} \mid h_{m n}^{-}$. Now we can prove a theorem which will contain Masley's result $h^{-}(K) \mid h^{-}(L)$ for cyclotomic fields $K=\mathbb{Q}\left(\zeta_{m}\right)$ and $L=\mathbb{Q}\left(\zeta_{m n}\right)$ as a special case: 
TheOREM 2. Let $K \subset L$ be $C M$-fields; then

$$
h^{-}(K)\left|h^{-}(L) \cdot\right| \kappa_{L / L^{+}} \mid \cdot \frac{\left(L \cap K^{1}: K\right)}{\left(L^{+} \cap\left(K^{+}\right)^{1}: K^{+}\right)},
$$

and the last quotient is a power of 2 .

Proof. Let $\nu_{K}$ and $\nu_{L}$ denote the norms $N_{K / K^{+}}$and $N_{L / L^{+}}$, respectively; then the following diagram is exact and commutative:

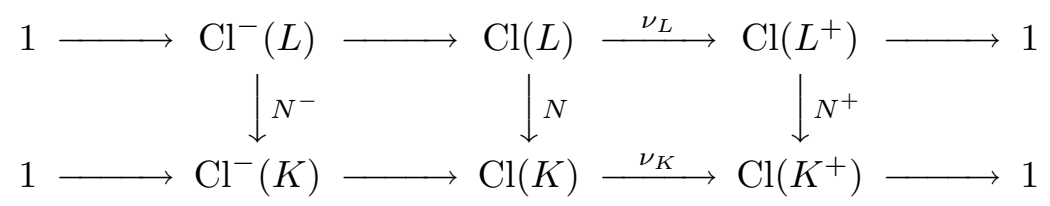

The snake lemma gives us an exact sequence

$$
1 \rightarrow \operatorname{ker} N^{-} \rightarrow \operatorname{ker} N \rightarrow \operatorname{ker} N^{+} \rightarrow \operatorname{cok} N^{-} \rightarrow \operatorname{cok} N \rightarrow \operatorname{cok} N^{+} \rightarrow 1 .
$$

Let $h(L / K)$ denote the order of ker $N$, and let $h^{-}(L / K)$ and $h\left(L^{+} / K^{+}\right)$be defined accordingly. The remark at the end of Section 1 shows

$$
|\operatorname{cok} N|=\left(L \cap K^{1}: K\right), \quad\left|\operatorname{cok} N^{+}\right|=\left(L^{+} \cap\left(K^{+}\right)^{1}: K^{+}\right) .
$$

The alternating product of the orders of the groups in exact sequences equals 1 , so the above sequence implies

$$
h^{-}(L / K) \cdot h\left(L^{+} / K^{+}\right) \cdot|\operatorname{cok} N|=h(L / K) \cdot\left|\operatorname{cok} N^{-}\right| \cdot\left|\operatorname{cok} N^{+}\right| .
$$

The exact sequence

$$
1 \rightarrow \operatorname{ker} N^{-} \rightarrow \mathrm{Cl}^{-}(L) \rightarrow \mathrm{Cl}^{-}(K) \rightarrow \operatorname{cok} N^{-} \rightarrow 1
$$

gives us

$$
h^{-}(L / K) \cdot h^{-}(K)=h^{-}(L) \cdot\left|\operatorname{cok} N^{-}\right| .
$$

Collecting everything we find that

$$
h^{-}(K) \cdot \frac{h(L / K)}{h\left(L^{+} / K^{+}\right)} \cdot \frac{\left(L^{+} \cap\left(K^{+}\right)^{1}: K^{+}\right)}{\left(L \cap K^{1}: K\right)}=h^{-}(L) .
$$

Now the claimed divisibility property follows if we can prove that $h\left(L^{+} / K^{+}\right)$ divides $h(L / K) \cdot\left|\kappa_{L / L^{+}}\right|$. But this is easy: exactly $h\left(L^{+} / K^{+}\right) /\left|\kappa_{L / L^{+}}\right|$ideal classes of ker $N^{+} \subset \mathrm{Cl}\left(L^{+}\right)$survive the transfer to $\mathrm{Cl}(L)$, and if the norm of $L^{+} / K^{+}$kills an ideal class $c \in \mathrm{Cl}\left(L^{+}\right)$, the same thing happens to the transferred class $c^{j}$ when the norm of $L / K$ is applied. We remark in passing that $\left|\kappa_{L / L^{+}}\right| \leq 2$ (see Hasse [H, Satz 18]).

It remains to show that $\left(L \cap K^{1}: K\right) /\left(L^{+} \cap\left(K^{+}\right)^{1}: K^{+}\right)$is a power of 2. Using induction on $(L: K)$, we see that it suffices to prove that if $L / K$ is an unramified abelian extension of CM-fields of odd prime degree $(L: K)=q$, then so is $L^{+} / K^{+}$. Suppose otherwise; then there exists a 
finite prime $\mathfrak{p}$ which ramifies, and since $L^{+} / K^{+}$is cyclic, $\mathfrak{p}$ has ramification index $q$. Now $L / K^{+}$is cyclic of order $2 q$, hence $K$ must be the inertia field of $\mathfrak{p}$, contradicting the assumption that $L / K$ is unramified. We conclude that $L^{+} / K^{+}$is also unramified, and so odd factors of $\left(L \cap K^{1}: K\right)$ cancel against the corresponding factors of $\left(L^{+} \cap\left(K^{+}\right)^{1}: K^{+}\right)$.

Corollary 1 ([LOO]). Let $K \subset L$ be CM-fields such that $(L: K)$ is odd; then $h^{-} K \mid h^{-}(L)$.

Proof. From $(*)$ and the fact that $\left(L^{+} \cap\left(K^{+}\right)^{1}: K^{+}\right)=1$ (this index is a power of 2 and divides ( $L: K)$, which is odd), we see that it is sufficient to show that $h\left(L^{+} / K^{+}\right) \mid h(L / K)$. This in turn follows if we can prove that no ideal class from $\operatorname{ker} N^{+} \subseteq \mathrm{Cl}\left(L^{+}\right)$capitulates when transferred to $\mathrm{Cl}(L)$. Assume therefore that $\kappa_{L / L^{+}}=\langle[\mathfrak{a}]\rangle$. If $w_{L} \equiv 2 \bmod 4$, then by Theorem 1(i.2) we may assume that $L=L^{+}(\sqrt{\alpha})$, where $\alpha \mathfrak{O}_{L^{+}}=\mathfrak{a}^{2}$. Since $(L: K)$ is odd, we can choose $\alpha \in O_{K^{+}}$, hence $N^{+}(\mathfrak{a})=\mathfrak{a}^{(L: K)}$ shows that the ideal class $[\mathfrak{a}]$ is not contained in $\operatorname{ker} N^{+}$. The proof in the case $w_{L} \equiv 0 \bmod 4$ is completely analogous.

Remark. For any prime $p$, let $\mathrm{Cl}_{p}^{-}(K)$ denote the $p$-Sylow subgroup of $\mathrm{Cl}^{-}(K)$; then $\mathrm{Cl}_{p}^{-}(K) \subseteq \mathrm{Cl}_{p}^{-}(L)$ for every $p \nmid(L: K)$. This is trivial, because ideal classes with order prime to $(L: K)$ cannot capitulate in $L / K$.

Corollary $2([\mathrm{MM}])$. If $K=\mathbb{Q}\left(\zeta_{m}\right)$ and $L=\mathbb{Q}\left(\zeta_{m n}\right)$ for some $m, n \in$ $\mathbb{N}$, then $h^{-}(K) \mid h^{-}(L)$.

Pr o o f. We have shown in Section 2 that $j_{K^{+} \rightarrow K}$ and $j_{L^{+} \rightarrow L}$ are injective in this case. Moreover, $L / K$ does not contain a non-trivial subfield of $K^{1}$ (note that $p$ is completely ramified in $L / K$ if $n=p$, and use induction).

The special case $m=p^{a}, n=p$ of Corollary 2 can already be found in [We]. Examples of CM-fields $L / K$ such that $h^{-}(K) \nmid h^{-}(L)$ have been given by Hasse $[\mathrm{H}]$; here are some more:

1. Let $d_{1} \in\{-4,-8,-q(q \equiv 3 \bmod 4)\}$ be a prime discriminant, and suppose that $d_{2}>0$ is the discriminant of a real quadratic number field such that $\left(d_{1}, d_{2}\right)=1$. Put $K=\mathbb{Q}\left(\sqrt{d_{1} d_{2}}\right)$ and $L=\mathbb{Q}\left(\sqrt{d_{1}}, \sqrt{d_{2}}\right)$; then $Q(L)=1$ and $\kappa_{L / L^{+}}=1$ by Example 4 , and $\left(L \cap K^{1}: K\right)=2 \cdot\left(L^{+} \cap\left(K^{+}\right)^{1}: K^{+}\right)$ since $L / K$ is unramified but $L^{+} / K^{+}$is not. The class number formula (1) below shows that in fact $h^{-}(K) \nmid h^{-}(L)$.

2. Let $d_{1}=-4, d_{2}=8 m$ for some odd $m \in \mathbb{N}$, and suppose that $2=$ $(2, \sqrt{2 m})$ is not principal in $\mathfrak{O}_{k}$, where $k=\mathbb{Q}(\sqrt{2 m})$. Then $h^{-}(K) \nmid h^{-}(L)$ for $K=\mathbb{Q}(\sqrt{-2 m}), L=\mathbb{Q}(\sqrt{-1}, \sqrt{2 m})$. Here $\left(L \cap K^{1}: K\right)=\left(L^{+} \cap\left(K^{+}\right)^{1}\right.$ : $\left.K^{+}\right)$, but $\kappa_{L / L^{+}}=\langle[2]\rangle$, since $2 \mathfrak{O}_{L}=(1+i)$. This example shows that we cannot drop the factor $\kappa_{L / L^{+}}$in Theorem 2 . 
Other examples can be found by replacing $d_{1}$ in Example 2 by $d_{1}=-8$ or $d_{2}$ by $d_{2}=4 m, m \in \mathbb{N}$ odd. The proof that in fact $h^{-}(K) \nmid h^{-}(L)$ for these fields uses Theorem 1, as well as Propositions 2 and 3 below.

4. Metsänkylä's factorization. An extension $L / K$ is called a $V_{4^{-}}$ extension of CM-fields if

1. $L / K$ is normal and $\operatorname{Gal}(L / K) \simeq V_{4}=(2,2)$;

2. Exactly two of the three quadratic subfields are CM-fields; call them $K_{1}$ and $K_{2}$, respectively.

This implies, in particular, that $K$ is totally real, and that $L$ is a CM-field with maximal real subfield $L^{+}=K_{3}$. We will write $Q_{1}=Q\left(K_{1}\right), W_{1}=W_{K_{1}}$, etc.

Louboutin [Lou, Prop. 13] has given an analytic proof of the following class number formula for $V_{4}$-extension of CM-fields, which contains Lemma 8 of Ferrero $[\mathrm{F}]$ as a special case:

Proposition 2. Let $L / K$ be a $V_{4}$-extension of $C M$-fields; then

$$
h^{-}(L)=\frac{Q(L)}{Q_{1} Q_{2}} \cdot \frac{w_{L}}{w_{1} w_{2}} h^{-}\left(K_{1}\right) h^{-}\left(K_{2}\right) .
$$

Proof. Kuroda's class number formula (for an algebraic proof see [L]) yields

$$
h(L)=2^{d-\kappa-2-v} q(L) h\left(K_{1}\right) h\left(K_{2}\right) h\left(L^{+}\right) / h(K)^{2},
$$

where

- $d=(K: \mathbb{Q})$ is the number of infinite primes of $K$ ramified in $L / K$;

- $\kappa=d-1$ is the $\mathbb{Z}$-rank of the unit group of $K$;

- $v=1$ if and only if all three quadratic subfields of $L / K$ can be written as $K(\sqrt{\varepsilon})$ for units $\varepsilon \in E_{K}$, and $v=0$ otherwise;

- $q(L)=\left(E_{L}: E_{1} E_{2} E_{3}\right)$ is the unit index for extensions of type $(2,2)$; here $E_{j}$ is the unit group of $K_{j}$ (similarly, let $W_{j}$ denote the group of roots unity in $L_{j}$ ).

Now we need to find a relation between the unit indices involved; we assert

Proposition 3. If $L / K$ is a $V_{4}$-extension of $C M$-fields, then

$$
\frac{Q(L)}{Q_{1} Q_{2}} \cdot \frac{w_{L}}{w_{1} w_{2}}=2^{-1-v} q(L) .
$$

Proof of Proposition 3. We start with the observation

$$
Q(L)=\left(E_{L}: W_{L} E_{3}\right)=\left(E_{L}: E_{1} E_{2} E_{3}\right) \frac{\left(E_{1} E_{2} E_{3}: W_{1} W_{2} E_{3}\right)}{\left(W_{L} E_{3}: W_{1} W_{2} E_{3}\right)} .
$$


In [L] we have defined groups $E_{j}^{*}=\left\{\varepsilon \in E_{j}: N_{j} \varepsilon\right.$ is a square in $\left.E_{K}\right\}$, where $N_{j}$ denotes the norm of $K_{j} / K$; we have also shown that

$$
\left(E_{1} E_{2} E_{3}: E_{1}^{*} E_{2}^{*} E_{3}^{*}\right)=2^{-v} \prod\left(E_{j}: E_{j}^{*}\right)
$$

and $E_{j} / E_{j}^{*} \simeq E_{K} / N_{j} E_{j}$. Now Proposition 1(a) gives $\left(E_{K}: N_{j} E_{j}\right)=Q_{j}$ for $j=1,2$, and we claim

1. $\left(W_{L} E_{3}: W_{1} W_{2} E_{3}\right)=\left(W_{L}: W_{1} W_{2}\right)=2 \cdot \frac{w_{L}}{w_{1} w_{2}} ;$
2. $E_{1}^{*} E_{2}^{*} E_{3}^{*}=W_{1} W_{2} E_{3}^{*} ;$

3. $\left(W_{1} W_{2} E_{3}: W_{1} W_{2} E_{3}^{*}\right)=\left(E_{3}: E_{3}^{*}\right)$.

This will give us

$$
Q(L)=2^{-1-v} q(L) Q_{1} Q_{2} \frac{w_{1} w_{2}}{w_{L}},
$$

completing the proof of Proposition 3; inserting (2) into equation (1) and recalling the definition of the minus class number yields Louboutin's formula.

We still have to prove the three claims above:

1. $W_{L} E_{3} / W_{1} W_{2} E_{3} \simeq W_{L} /\left(W_{L} \cap W_{1} W_{2} E_{3}\right) \simeq W_{L} / W_{1} W_{2}$, and the claim follows from $W_{1} \cap W_{2}=\{-1,+1\}$;

2. We only need to show that $E_{1}^{*} E_{2}^{*} E_{3}^{*} \subset W_{1} W_{2} E_{3}^{*}$; but Proposition 1(a) shows that $\varepsilon \in E_{1}^{*} \Leftrightarrow \varepsilon^{\sigma+1} \in E_{K}^{2} \Leftrightarrow \varepsilon \in W_{1} E_{K}$, and this implies the claim;

3. $W_{1} W_{2} E_{3} / W_{1} W_{2} E_{3}^{*} \simeq E_{3} / E_{3} \cap W_{1} W_{2} E_{3}^{*} \simeq E_{3} / E_{3}^{*}$.

Combining the result of Section 3 with Proposition 2, we get the following

Theorem 3. Let $L_{1}$ and $L_{2}$ be CM-fields, and let $L=L_{1} L_{2}$ and $K=L_{1}^{+} L_{2}^{+}$; then $L / K$ is a $V_{4}$-extension of $C M$-fields with subfields $K_{1}=$ $L_{1} L_{2}^{+}, K_{2}=L_{1}^{+} L_{2}, K_{3}=L^{+}$, and

$$
h^{-}(L)=\frac{Q(L)}{Q_{1} Q_{2}} \cdot \frac{w_{L}}{w_{1} w_{2}} h^{-}\left(L_{1}\right) h^{-}\left(L_{2}\right) T_{1} T_{2},
$$

where $T_{1}=h^{-}\left(L_{1} L_{2}^{+}\right) / h^{-}\left(L_{1}\right)$ and $T_{2}=h^{-}\left(L_{2} L_{1}^{+}\right) / h^{-}\left(L_{2}\right)$.

If we assume that $\kappa_{1}=\kappa_{2}=1$ ( $\kappa_{1}$ is the group of ideal classes capitulating in $L_{1} L_{2}^{+} / K$ and $\kappa_{2}$ is defined similarly) and that

$$
\begin{aligned}
& \left(L_{1} L_{2}^{+} \cap L_{1}^{1}: L_{1}\right)=\left(L_{1}^{+} L_{2}^{+} \cap\left(L_{1}^{+}\right)^{1}: L_{1}^{+}\right), \\
& \left(L_{2} L_{1}^{+} \cap L_{2}^{1}: L_{2}\right)=\left(L_{2}^{+} L_{1}^{+} \cap\left(L_{2}^{+}\right)^{1}: L_{2}^{+}\right),
\end{aligned}
$$

then $T_{1}$ and $T_{2}$ are integers.

Pr o of. Theorem 3 follows directly from Theorem 2 and Proposition 2. 
The following Hasse diagram explains the situation:

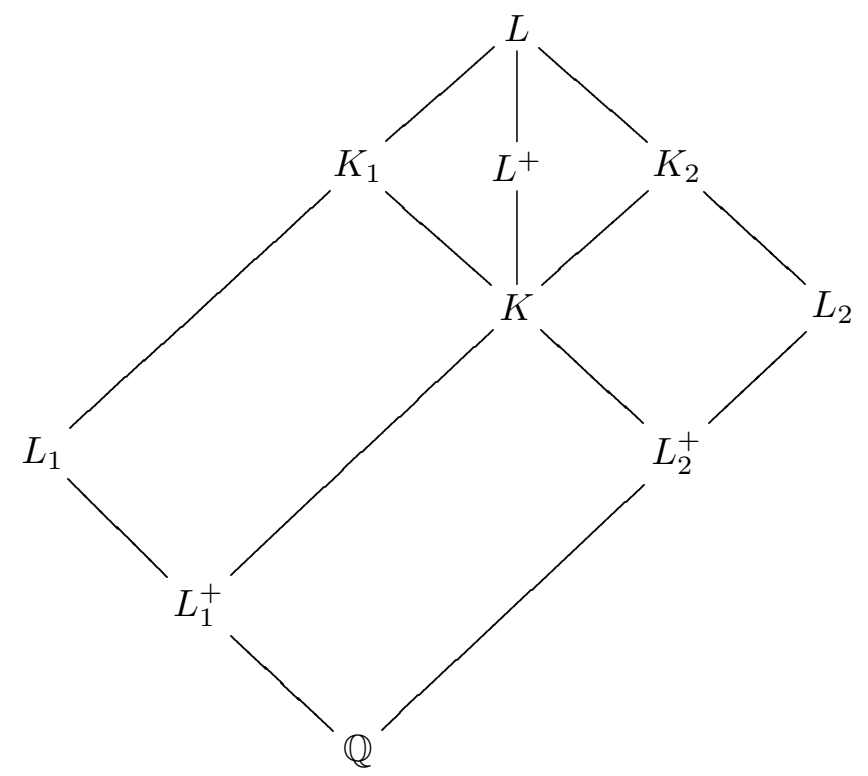

Now let $m=p^{\mu}$ and $n=q^{\nu}$ be prime powers, and suppose that $p \neq q$. Moreover, let $L_{1} \subseteq \mathbb{Q}\left(\zeta_{m}\right)$ and $L_{2} \subseteq \mathbb{Q}\left(\zeta_{n}\right)$ be CM-fields. Then

(1) $Q(L)=2, Q_{1}=Q\left(L_{1} L_{2}^{+}\right)=Q_{2}=Q\left(L_{2} L_{1}^{+}\right)=1$ : this has been proved in Proposition 1(h) and Example 4 in Section 2;

(2) $w_{1} w_{2}=2 w_{L}$ (obviously);

(3) $\kappa_{1}=\kappa_{2}=1$ : see Example 4 in Section 2;

(4) $\left(L_{1} L_{2}^{+} \cap L_{1}^{1}: L_{1}\right)=\left(L_{1}^{+} L_{2}^{+} \cap\left(L_{1}^{+}\right)^{1}: L_{1}^{+}\right)$: this, as well as the corresponding property for $K_{2}$, is obvious, because the prime ideals above $p$ and $q$ ramify completely in $L / L_{2}$ and $L / L_{1}$, respectively.

In particular, we have the following

Corollary $([\mathrm{M}])$. Let $L_{1} \subseteq \mathbb{Q}\left(\zeta_{m}\right)$ and $L_{2} \subseteq \mathbb{Q}\left(\zeta_{n}\right)$ be CM-fields, where $m=p^{\mu}$ and $n=q^{\nu}$ are prime powers, and let $L=L_{1} L_{2}$; then

$$
h^{-}(L)=h^{-}\left(L_{1}\right) h^{-}\left(L_{2}\right) T_{1} T_{2},
$$

where $T_{1}=h^{-}\left(L_{1} L_{2}^{+}\right) / h^{-}\left(L_{1}\right)$ and $T_{2}=h^{-}\left(L_{2} L_{1}^{+}\right) / h^{-}\left(L_{2}\right)$ are integers.

It still remains to identify the character sums $T_{01}$ and $T_{10}$ in $[\mathrm{M}]$ with the class number factors $T_{1}$ and $T_{2}$ given above. But this is easy: the character group $X\left(L_{1}\right)$ corresponding to the field $L_{1}$ is generated by a character $\chi_{1}$, and it is easily seen that 


$$
\begin{aligned}
X\left(L_{1}\right) & =\left\langle\chi_{1}\right\rangle, & X\left(L_{1} L_{2}^{+}\right) & =\left\langle\chi_{1}, \chi_{2}^{2}\right\rangle, \\
X\left(L_{2}\right) & =\left\langle\chi_{2}\right\rangle, & X\left(L_{2} L_{1}^{+}\right) & =\left\langle\chi_{2}, \chi_{1}^{2}\right\rangle, \\
X(L) & =\left\langle\chi_{1}, \chi_{2}\right\rangle, & X\left(L^{+}\right) & =\left\langle\chi_{1} \chi_{2}, \chi_{1}^{2}\right\rangle .
\end{aligned}
$$

The analytical class number formula for an abelian CM-field $K$ reads

$$
h^{-}(K)=Q(K) w_{K} \prod_{\chi \in X^{-}(K)} \frac{1}{2 \mathfrak{f}(\chi)} \sum_{a \bmod \mathfrak{m}^{+}(\chi)}(-\chi(a) a),
$$

where $a \bmod ^{+} \mathfrak{f}(\chi)$ indicates that the sum is extended over all $1 \leq a \leq \mathfrak{f}(\chi)$ such that $(a, \mathfrak{f}(\chi))=1$, and $X^{-}(L)=X(L) \backslash X\left(L^{+}\right)$is the set of $\chi \in X(L)$ such that $\chi(-1)=-1$. Applying formula (3) to the CM-fields listed above and noting that $Q(L)=2, Q\left(L_{1}\right)=Q\left(L_{2}\right)=Q\left(L_{1} L_{2}^{+}\right)=Q\left(L_{2} L_{1}^{+}\right)=1$ and $2 w_{L}=w_{1} w_{2}$, we find

$$
h^{-}(L)=h^{-}\left(L_{1}\right) \cdot h^{-}\left(L_{2}\right) \prod_{\chi \in X^{*}(L)} \frac{1}{2 \mathfrak{f}(\chi)} \sum_{a \bmod +\mathfrak{f}(\chi)}(-\chi(a) a),
$$

where $X^{*}(L)$ is the subset of all $\chi \in X^{-}(L)$ not lying in $X^{-}\left(L_{1}\right)$ or $X^{-}\left(L_{2}\right)$. Now define $X_{1}(L)=\left\{\chi=\chi_{1}^{x} \chi_{2}^{y} \in X^{*}(L): x \equiv 1 \bmod 2, y \equiv 0 \bmod 2\right\}$, and let $X_{2}(L)$ be defined accordingly. Then $X^{*}(L)=X_{1}(L) \cup X_{2}(L)$, and

$$
h^{-}\left(L_{1}\right) \cdot \prod_{\chi \in X_{1}(L)} \frac{1}{2 \mathfrak{f}(\chi)} \sum_{a \bmod +\mathfrak{f}(\chi)}(-\chi(a) a)=h^{-}\left(L_{1} L_{2}^{+}\right),
$$

and we have shown that

$$
T_{1}=\prod_{\chi \in X_{1}(L)} \frac{1}{2 \mathfrak{f}(\chi)} \sum_{a \bmod +\mathfrak{f}(\chi)}(-\chi(a) a) .
$$

Comparing with the definition of Metsänkylä's factor $T_{10}$, this shows that indeed $T_{1}=T_{10}$.

Acknowldegements. I would like to thank Stéphane Louboutin and Ryotaro Okazaki for several helpful suggestions and for calling my attention to the papers of Horie and Uchida.

\section{References}

[F] B. Ferrero, The cyclotomic $\mathbb{Z}_{2}$-extension of imaginary quadratic number fields, Amer. J. Math. 102 (1980), 447-459.

[H] H. Hasse, Über die Klassenzahl abelscher Zahlkörper, Springer, Berlin, 1985.

[HY] M. Hirabayashi and K. Yoshino, Remarks on unit indices of imaginary abelian number fields, Manuscripta Math. 60 (1988), 423-436.

[Ho] K. Horie, On a ratio between relative class numbers, Math. Z. 211 (1992), 505521. 
[L] F. Lemmermeyer, Kuroda's class number formula, Acta Arith. 66 (1994), 245260.

[Lou] S. Louboutin, Determination of all quaternion octic CM-fields with class number 2, J. London Math. Soc., to appear.

[LOO] S. Louboutin, R. Okazaki and M. Olivier, The class number one problem for some non-abelian normal CM-fields, preprint, 1994.

[M] T. Metsänkylä, Über den ersten Faktor der Klassenzahl des Kreiskörpers, Ann. Acad. Sci. Fenn. Ser. A I 416, 1967.

[MM] J. M. Masley and H. L. Montgomery, Cyclotomic fields with unique factorization, J. Reine Angew. Math. 286/287 (1976), 248-256.

[O] R. Okazaki, On evaluation of L-functions over real quadratic fields, J. Math. Kyoto Univ. 31 (1991), 1125-1153.

[S] A. Scholz, Über die Lösbarkeit der Gleichung $t^{2}-D u^{2}=-4$, Math. Z. 39 (1934), 95-111.

[U] K. Uchida, Imaginary quadratic number fields with class number one, Tôhoku Math. J. 24 (1972), 487-499.

[W] L. Washington, Introduction to Cyclotomic Fields, Springer, New York, 1982.

[We] J. Westlund, On the class number of the cyclotomic number field, Trans. Amer. Math. Soc. 4 (1903), 201-212.

ERWIN-ROHDE-STR. 19

D-69120 HEIDELBERG, GERMANY

E-mail: HB3@IX.URZ.UNI-HEIDELBERG.DE 